Schmerz 2010 $24: 315-316$

DOI 10.1007/s00482-010-0945-8

Online publiziert: 28. Juli 2010

(c) Deutsche Gesellschaft zum Studium des Schmerzes. Published by Springer

Medizin Verlag - all rights reserved 2010

\author{
W. Meißner \\ Klinik für Anästhesiologie und Intensivtherapie, Friedrich-Schiller-Universität, Jena
}

\title{
Qualitätssicherung in der Schmerztherapie
}

ten, die für eine externe Qualitätssicherung verwendet werden sollen, sind wesentlich umfangreicher als für eine interne (Routine-)Dokumention wie in der vorliegenden Publikation. So sollte beispielsweise die Erfassung der Schmerzintensität unter identischen Bedingungen (hinsichtlich Fremd-/Selbstbeurteilung, Erhebungsperson und -bedingungen) erfolgen, wenn die Daten multizentrisch verglichen werden sollen.

- Bedienerfreundlichkeit vs. Datenqualität: Die Autoren schlagen eine voreingestellte „1-Klick-Dokumentation“ für „Routinevisiten“ vor („schmerzfrei und zufrieden“ Feld VAS wird auf o gesetzt). Diese Voreinstellung wurde in $39 \%$ aller ausgewerteten Visiten verwendet, d. h. $39 \%$ aller Patienten wurde eine Schmerzintensität von „o “ zugeordnet - ein fantastisches Ergebnis, das in der Literatur bisher kaum jemals erreicht wurde. Mag im Rahmen der Routinedokumentation eine solche Erleichterung eventuell akzeptabel erscheinen, sind derartige Verfahren im Bereich der Qualitätssicherung äußerst kritisch zu beurteilen.

- Qualitätsbeurteilung durch den Beurteilten: Die Erhebung von Daten zur Qualitätssicherung durch diejenigen, deren Qualität überprüft werden soll, kann problematisch sein.

- Externe Qualitätssicherung sollte kompatibel sein mit konsentierten Kerndatensätzen bzw. existierenden Projekten (z. B. dem Netzwerk Regionalanästhesie des AK Regionalanästhesie der DGAI); für eine interne medizinische Dokumentation ist dies nicht zwangsläufig notwendig.
- Vollerfassung vs. Stichprobe: Medizinische Dokumentationen wie das vorgestellte Projekt müssen ausnahmslos alle Patienten abbilden (Vollerfassung), für die Ziele Leistungsdokumentation und Qualitätssicherung würde auch eine repräsentative Stichprobe genügen.

Diese wenigen Beispiele verdeutlichen die potenziellen Konflikte, die bei der - prinzipiell wünschenswerten - Nutzung einmal erhobener Daten für unterschiedliche Ziele entstehen können. Es gibt jedoch weitere Kontroversen auf dem Feld der Qualitätssicherung:

Der Deutsche vertraut kaum einer Institution so wie dem TÜV. Folgerichtig wurde auch die Qualitätssicherung in der Medizin „TÜVisiert“, d. h. auf Strukturund Prozessüberprüfung fokussiert. Diese Parameter sind zwar relativ leicht $-\mathrm{z}$. B. im Rahmen einer Zertifizierung - erfassbar, besitzen häufig aber nur Surrogatcharakter für die Ergebnisqualität. So konnte gezeigt werden, dass die breite Einführung des Prozesses „Schmerzmessung als 5. Vitalzeichen" keinerlei Verbesserung der Patientenversorgung zur Folge hatte [3]. Dies heißt nicht, dass ein solcher Prozess sinnlos ist, es bedeutet aber, dass er sich nicht als Qualitätsindikator eignet. Qualität aus Patientenperspektive erschließt sich eben nur durch eine einheitliche und valide Erhebung von für das Behandlungsergebnis relevanten Ergebnisparametern [4]. Was ist aber Ergebnisqualität in der Schmerztherapie?

Traditionell wird die Qualität der Schmerztherapie primär am Parameter Schmerzintensität gemessen und das Erreichen bzw. Unterschreiten bestimmter Cut-off-Werte propagiert. Eine aus- 
schließliche Orientierung des Therapieerfolges an (einer möglichst niedrigen) Schmerzintensität kann jedoch zu einer unangebrachten Einschätzung der Gesamtqualität und/oder einer Fehlsteuerung der Therapie führen: Ist es „gute "Schmerztherapie, wenn der Patient schmerzfrei, aber emetisch, kognitiv oder motorisch beeinträchtigt ist und das Bett nicht verlassen kann? In dem von Czaplik et al. vorgestellten Dokumentationssystem werden neben der Schmerzintensität erfreulicherweise auch Therapienebenwirkungen erfasst. Es wäre zu diskutieren, ob zusätzlich auch „funktionellere“ Parameter wie schmerzbedingte Beeinträchtigungen von Körperfunktionen (Mobilisierung, Ernährung) oder sogar Kenngrößen der postoperativen Rehabilitation wichtige Ergebnisitems sein könnten und ergänzend (vielleicht auch stichprobenartig) erhoben werden sollten.

Hieran wird deutlich, dass Qualitätssicherung (nicht nur in der Schmerztherapie) nie als isolierter Teilprozess betrachtet werden darf oder sich nur auf das Sammeln von Daten beschränken sollte, sondern sich an integrierenden Ergebnisparametern orientieren muss. In der postoperativen Schmerztherapie könnte dies beispielsweise so formuliert werden: Ziel ist eine Minimierung der postoperativen Komplikationen, funktionellen Beeinträchtigungen und Schmerzen, resultierend in einer frühzeitigen Entlassung eines zufriedenen Patienten.

Werkzeuge zur Messung von Qualitätsindikatoren sind nur dann hilfreich, wenn sie dem Nutzer Anhaltspunkte („Benchmarks") über die Bewertung seiner Ergebnisse liefern. Dass sich dazu fixierte Cut-off-Werte nur bedingt eignen, wurde bereits oben ausgeführt. Dagegen wird ein zeitlicher Vergleich mit den eigenen oder ein externer Vergleich mit den Messwerten anderer Akteure oft als hilfreich empfunden. „Benchmarking“ im erweiterten Sinn umfasst jedoch darüber hinaus die Möglichkeit des „Lernens vom Besten“ bzw. die Identifikation von „best practice“ z. B. durch kollegialen Austausch („peer auditing“) - denn nur wenn Konsequenzen aus den gemessenen Qualitätsdaten gezogen werden, kann es zu einer Qualitätsverbesserung kommen.
Das von Czaplik et al. vorgestellte Erfassungstool kann ein erster Schritt in diese Richtung sein, wenn es unter Beachtung der oben genannten Aspekte von Routinedokumentation und Qualitätssicherung in ein umfassenderes Konzept eingegliedert werden kann. Manchmal gilt aber auch: Weniger ist mehr!

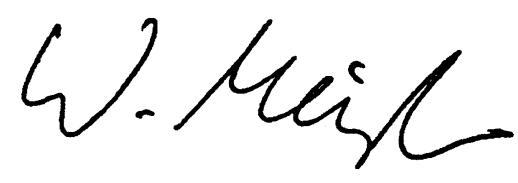

W. Meißner

\section{Korrespondenzadresse \\ PD Dr. W. Meißner \\ Klinik für Anästhesiologie \\ und Intensivtherapie, \\ Friedrich-Schiller-Universität \\ Erlanger Allee 101, 07747 Jena \\ Winfried.Meissner@med.uni-jena.de}

Interessenkonflikt. Der korrespondierende Autor weist auf folgende Beziehungen hin: Der Autor ist Koordinator der Qualitätssicherungsprojekte QUIPS und PAIN-OUT.

\section{Literatur}

1. Czaplik et al (2010) Qualitätssicherung in der Akutschmerztherapie. Schmerz 24:358-366

2. Heller G et al. (2008) Perspektiven der Qualitätssicherung mit Routinedaten. In: Wiss Institut der AOK (Hrsg) Krankenhausreport 2007. Schattauer, Stuttgart, S 171-184

3. Mularski RA et al. (2006) Measuring pain as the 5 th vital sign does not improve quality of pain management. J Gen Intern Med 21:607-612

4. Gordon DB et al (2005) American Pain Society recommendations for improving the quality of acute and cancer pain management. Arch Intern Med 165:1574-1580

\section{Symposium „Neue Konzepte zum Muskelschmerz: Grundlage und Klinik"}

Mainz, 17.-18. September 2010

Liebe Kolleginnen und Kollegen, wir dürfen Sie ganz herzlich zu unserem Schmerzsymposium „Neue Konzepte zum Muskelschmerz: Grundlage und Klinik" am 17.-18. September 2010 in Mainz, Favorite Parkhotel, einladen.

Hervorragende Referenten aus dem In- und Ausland werden dieses höchst aktuelle Thema von allen Seiten mit interessanten Beiträgen und Diskussionen höchst professionell gestalten.

Wir wissen noch immer zu wenig über die muskuloskelettalen Schmerzen obwohl es sich um die häufigste Ursache bei muskuloskelettalen Beschwerden, insbesondere auch bei Chronifizierungsvorgängen handelt. Fragen wie: Wie kommt es zu Muskelschmerzen? Warum haben wir ein so großes Chronifizierungspotential? Wie sauber können wir die Diagnose abgrenzen gegenüber arthrogenen und neurogenen Ursachen? Wie sieht unser neues Behandlungskonzept aus?

Fragen wie diese beschäftigen uns alle sehr und trotzdem wird selten darauf eingegangen. Dieses Symposium will auf jeden Fall hier einen Anfang machen.

Wir würden uns freuen, wenn Sie von dieser Möglichkeit Gebrauch machen und wir Sie Mitte September in Mainz begrüßen dürfen.

Mit herzlichen Grüßen

Ihr

S. Mense

H.-R. Casser

Weitere Details finden Sie im Kongresskalender am Ende dieser Ausgabe. 